\title{
Structure and Spatial Distribution of Ge Nanocrystals Subjected to Fast Neutron Irradiation
}

\author{
Regular Paper
}

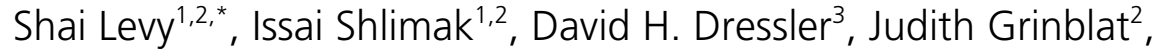 \\ Yossi Gofer ${ }^{3}$, Tiecheng Lu $\mathrm{Lu}^{4}$ and Alexander N. Ionov ${ }^{5}$ \\ 1 Jack and Pearl Redneck Institute of Advanced Technology, Bar-Ilan University, Israel \\ 2 Bar-llan Institute of Nanotechnology and Advanced Materials, Bar-Ilan University, Israel \\ 3 Department of Chemistry, Bar Ilan University, Israel \\ 4 Department of Physics and Key Laboratory for Radiation Physics Technology of Ministry of Education, Sichuan University, P.R. China \\ 5 A. F. Ioffe Physico-Technical Institute RAS, Russia \\ *Corresponding author E-mail: shai.levy10@gmail.com
}

Received 5 April 2011; Accepted 5 May 2011

\begin{abstract}
The influence of fast neutron irradiation on the structure and spatial distribution of Ge nanocrystals (NC) embedded in an amorphous $\mathrm{SiO}_{2}$ matrix has been studied. The investigation was conducted by means of laser Raman Scattering (RS), High Resolution Transmission Electron Microscopy (HR-TEM) and X-ray photoelectron spectroscopy (XPS). The irradiation of GeNC samples by a high dose of fast neutrons lead to a partial destruction of the nanocrystals. Full reconstruction of crystallinity was achieved after annealing the radiation damage at $800^{\circ} \mathrm{C}$, which resulted in full restoration of the RS spectrum. HR-TEM images show, however, that the spatial distributions of Ge-NC changed as a result of irradiation and annealing. A sharp decrease in NC distribution towards the $\mathrm{SiO}_{2}$ surface has been observed. This was accompanied by XPS detection of Ge oxides and elemental Ge within both the surface and subsurface region.
\end{abstract}

Keywords Ge nanocrystals, Neutron radiation effects, Structure and spatial distribution.

\section{Introduction}

Since the early 1990s, samples of Si and Ge nanocrystals (NC-Si, Ge-NC) embedded in a silicon dioxide $\left(\mathrm{SiO}_{2}\right)$ matrix have attracted much interest due to strong visible photoluminescence at room temperature [1,2]. Additional electrical studies performed on these NC have revealed charge retention properties [3-14], suggesting the future incorporation of such structures in silicon-based electronic technology such as optoelectronic and microelectronic devices [5, 6, 8-16]. It has been shown [17, 18] that Ge-NC's smaller band-gap makes it a more suitable material than NC-Si for silicon-based technology. This property has made Ge-NC the promising candidate for the creation of a new generation of nano-scale optoelectronic devices. In this respect, investigation of the ability of the material to withstand different destructive influences is important for the design of semiconductor devices that can normally operate in a radiation-rich environment.

Early work investigated the role of ion-induced damage to the structure of Ge-NC. It was observed that Ge-NC 
are highly sensitive and become amorphous at a $\mathrm{Si}^{+}$ion dose of $5 \times 10^{13} \mathrm{~cm}^{-2}$ [19]. Irradiation by high energy neutrons in a nuclear reactor leads to different structure and distribution of the radiation damage, because neutrons are not charged and have significantly lighter mass when compared to ions. Clash with fast neutrons with energy up to several $\mathrm{MeV}$ causes displacement of atoms in the lattice in cascades, which produce radiation damage in the form of nanometer size amorphous clusters, impurity-vacancy complexes, etc. [20]. These directly contribute to the destruction of the optical and electrical performance of bulk $\mathrm{Si}$ and Ge-based devices (such as diodes and hetero-junction bipolar transistors (HBTs) [21]). MOS (Metal Oxide Semiconductor) devices are known to withstand displacement-caused degradation only up to integral dose of $10^{15}$ neutrons $/ \mathrm{cm}^{2}$ [22]. In this investigation, Ge-NC samples were subjected to a much higher dose of $10^{20}$ neutrons $/ \mathrm{cm}^{2}$.

In addition, besides the radiation damage due to fast neutrons, absorption of slow (thermal) neutrons leads to transmutation of different isotopes of Ge into impurities due to nuclear reactions. For example, ${ }^{74} \mathrm{Ge}$ transforms to ${ }^{75} \mathrm{As}$ (donor impurity), ${ }^{70} \mathrm{Ge}$ into ${ }^{71} \mathrm{Ga}$ (acceptor impurity) and ${ }^{76} \mathrm{Ge}$ into ${ }^{77} \mathrm{Se}$ (deep double donor impurity). This process is called "neutron transmutation doping" (NTD). Concentration of NTD introduced impurities is proportional to abundance of the given isotope in the natural Ge composition, cross-section of the absorption of thermal neutron and the integral dose of thermal neutron flux $[20,23]$. Transmutation processes are accompanied by emission of $\gamma$-quanta and $\beta$-particles, which can, in principle, contribute to the radiation damage as well. Investigation of bulk NTD-Ge showed that almost all radiation damages are removed after annealing of neutron irradiated samples at $400^{\circ} \mathrm{C}[24]$.

The Ge-NC samples investigated in this work were obtained in cooperation with the group from Sichuan University, People's Republic of China. Investigations of this group [2528] were focused on the influence of ${ }^{75} \mathrm{As}$ impurities introduced by NTD into samples prepared by ion implantation of ${ }^{74} \mathrm{Ge}$ into $\mathrm{SiO}_{2}$ matrix. The aim of this work is to investigate the structure, size and spatial distribution of Ge-NC in samples after all steps of treatment: fabrication, neutron irradiation and radiation damage annealing.

\section{Experimental details}

Our samples underwent five stages of treatments described below; at each stage of treatment a control sample was kept.

The Ge-NC samples were prepared using ${ }^{74} \mathrm{Ge}^{+}$ionimplantation into a $500 \mathrm{~nm}$ thick amorphous $\mathrm{SiO}_{2}$ layer, deposited on a silicon substrate with $<100>$ oriented surface. The details of the ion-implantation are documented in $[25,26]$. The $\mathrm{Ge}^{+}$ions were accelerated to $150 \mathrm{keV}$, with a dose of $1 \times 10^{17}$ ions $/ \mathrm{cm}^{2}$. These samples were labeled as "implanted". A second treatment was conducted on the implanted samples by annealing at $800^{\circ} \mathrm{C}$ for $1 \mathrm{~h}$. As a result, the randomly distributed Ge atoms formed nanocrystals (NC) [29]. These samples were labeled as "Ge-NC". The third process consisted of subjecting the Ge-NC samples to an intensive neutron irradiation in a research nuclear reactor, with the integral dose of $10^{20}$ neutrons $/ \mathrm{cm}^{2}$, and subsequently labeled as "irradiated". The neutron irradiation was performed at room-temperature. After irradiation, a fourth group consisting of irradiated Ge-NC samples was annealed at $600^{\circ} \mathrm{C}$ for $1 \mathrm{~h}$ to remove the radiation damage. These samples were labeled as "semifinal". The remaining samples were annealed a second time at $800^{\circ} \mathrm{C}$ for $1 \mathrm{~h}$. This fifth group of samples was labeled as "final".

It should be noted that to distinguish between defects, resulting from the destruction of the lattice by the fast neutron irradiation, and elements and defects that appear due to transmutation and the presence of impurities, half of the irradiated samples were irradiated within a cadmium (Cd) box, which was used as a shield from the slow (thermal) neutrons. As a result, samples irradiated within a Cd box were subjected only to the influence of fast neutrons, while samples irradiated outside a $\mathrm{Cd}$ box were subjected to the flux of both fast and slow neutrons. We note that in our study of Ge-NC structure, no significant differences were found between the samples irradiated inside and outside a $\mathrm{Cd}$ box, therefore in the following, we will not distinguish between these two types of sample. The influence connected with transmutation doping may be detected in a study of the electrical and optical properties of the samples, which is outside the scope of this manuscript.

Measurements of Raman scattering (RS) and High Resolution Transmission Electron Microscopy (HR-TEM) are usually used to confirm the crystalline structure. Crystalline $\mathrm{Ge}$ is characterized by the Raman peak centered at approximately $300 \mathrm{~cm}^{-1}$, which corresponds to the optical phonon frequency in bulk Ge crystals [30]. Measurements of HR-TEM images enable the examination of fine details of the NC structure. In addition to the crystalline structure analysis, the surfaces of the samples were analyzed by X-ray photoelectron spectroscopy (XPS).

RS spectra were measured with a Raman microscope LabRam HR and were taken in the back-scattering geometry at room-temperature using a $514.5 \mathrm{~nm}$ laser source for excitation. HR-TEM images were obtained using the $200 \mathrm{kV}$ JEOL, JEM 2100 HR-TEM (LaB6) integrated with a scanning device comprising annular 
dark-field and bright-field detectors and with a Noran System Six EDS (energy dispersive X-ray spectroscopy) system for elemental analysis. For cross-section imaging, HR-TEM samples were prepared by a focused ion beam (FIB) lift-out technique. XPS measurements were carried out on AXIS HS Kratos Analytical electron spectrometer system. The spectra were acquired using a monochromatic Al-K (1486.6 eV) X-ray source.

\section{Results and discussion}

\subsection{Initial samples (before neutron irradiation)}

Curve $a$ in Fig. 1 shows RS spectra in the implanted sample, where Ge atoms do not yet form nanocrystals.

One can see a broad asymmetric unstructured band which can be attributed to separate Ge atoms or amorphous nano-clusters [29, 33].

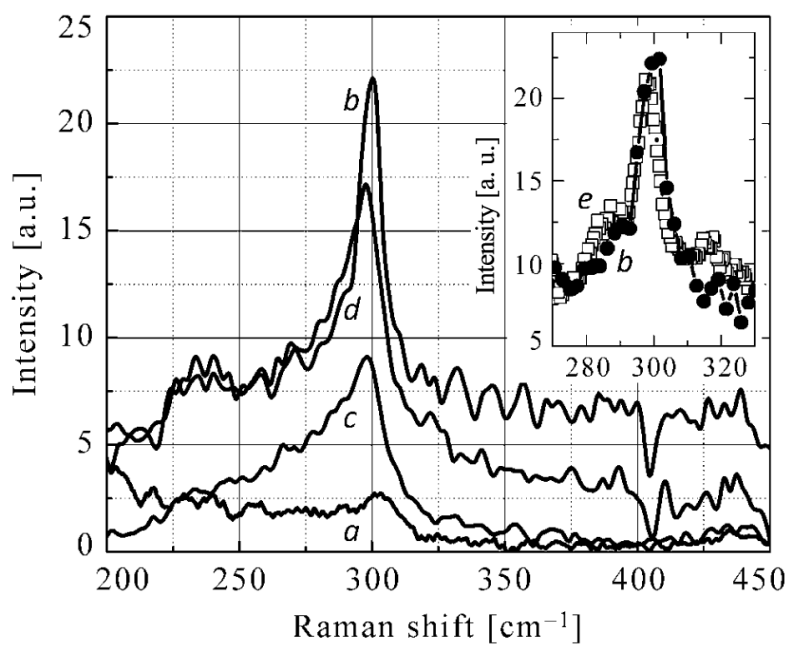

Figure 1. Raman spectra of (a) implanted, (b) Ge-NC, (c) irradiated and $(d)$ semifinal samples; inset displays RS around $300 \mathrm{~cm}^{-1}$ for Ge-NC (b) and final (e) samples.

Fig. $2 a$ shows bright-field HR-TEM cross-sectional image of a Ge-implanted sample, where Ge atoms have not yet formed nanocrystals. In bright-field mode it is possible to distinguish between the Ge clusters and the $\mathrm{SiO}_{2}$ matrix due to the contrast of the image, i.e. regions with $\mathrm{Ge}$ atoms appear darker while the $\mathrm{SiO}_{2}$ matrix appears brighter.

Nonappearance of NC is confirmed by the absence of a diffraction pattern.

Conforming to the SRIM simulation (Stopping and Range of Ions in Matter simulation) [32], the projection range and straggle (half-width of the distribution) for Ge ions with energy of $150 \mathrm{keV}$ implanted in $\mathrm{SiO}_{2}$ are approximately $100 \pm 5 \mathrm{~nm}$ and $30 \mathrm{~nm}$, respectively, which concur with the experimental observation (Fig. 2a).
In order to form nanocrystals, implanted samples were annealed at $800^{\circ} \mathrm{C}$. Formation of Ge-NC was confirmed by the appearance of the main RS peak, centered at $300 \mathrm{~cm}^{-1}$ (see curve $b$ in Fig. 1) which is characteristic of crystalline Ge. The absence of a significant shift of the Raman peak from $300 \mathrm{~cm}^{-1}$ indicates that no effects connected with stress [33] or quantum confinements [34] are observed. In addition, crystalline structure was observed in the enlarged HR-TEM image in the form of equidistant lattice planes (Fig. 2b). The HR-TEM image shows that Ge-NC regions have an almost spherical shape with a diameter of approximately 2-10 $\mathrm{nm}$. Inspection of the image reveals a correlation between the diameter and the depth of NC below the $\mathrm{SiO}_{2}$ surface: within the projection range (100 $\mathrm{nm})$, where the density of the implanted Ge atoms is maximal, the average diameter of Ge-NC is also largest and symmetrically decreases on both sides.
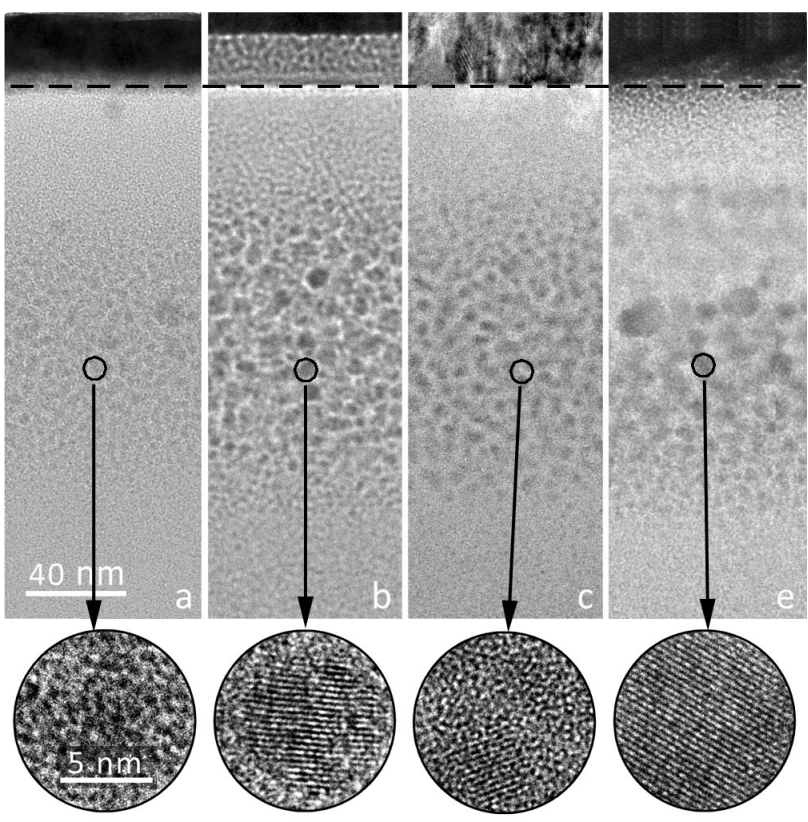

Figure 2. HR-TEM cross-section of (a) implanted (b) Ge-NC, (c) irradiated and (e) final samples. The dashed line indicates the surface of the samples covered by Pt which is needed for TEM cross-section preparation.

The composition of Ge-NC was studied by an accompanying investigation of selected area electron diffraction (SAED) pattern of the Ge-NC layer inside the $\mathrm{SiO}_{2}$ matrix and live fast Fourier transform (FFT) performed on an HR-TEM image of a single NC (Fig. 3). In SAED measurements, the d-spacing between planes (111), (022) and (222) was found to be 3.2, 1.95 and $1.67 \AA$ $\pm 0.04 \AA$, respectively. In the case of FFT the d-spacing between planes (111), (022) and (-111) was found to be 3.3, 2.0 and $3.4 \AA \pm 0.06 \AA$ respectively.

Using both diffraction and live FFT, it is possible to conclude that the crystals consist of Ge and are not a mix of any compound of Ge. It has also been observed that 
different crystallographic orientations coexist in some single NC, which can be explained by the existence of more than one nucleation center that serve as seeds forming each Ge-NC. That is, nucleation centers form small sized NC during the annealing process. These NC aggregate toward one another, and once they come into contact their lattice planes start to adjust at the interface.

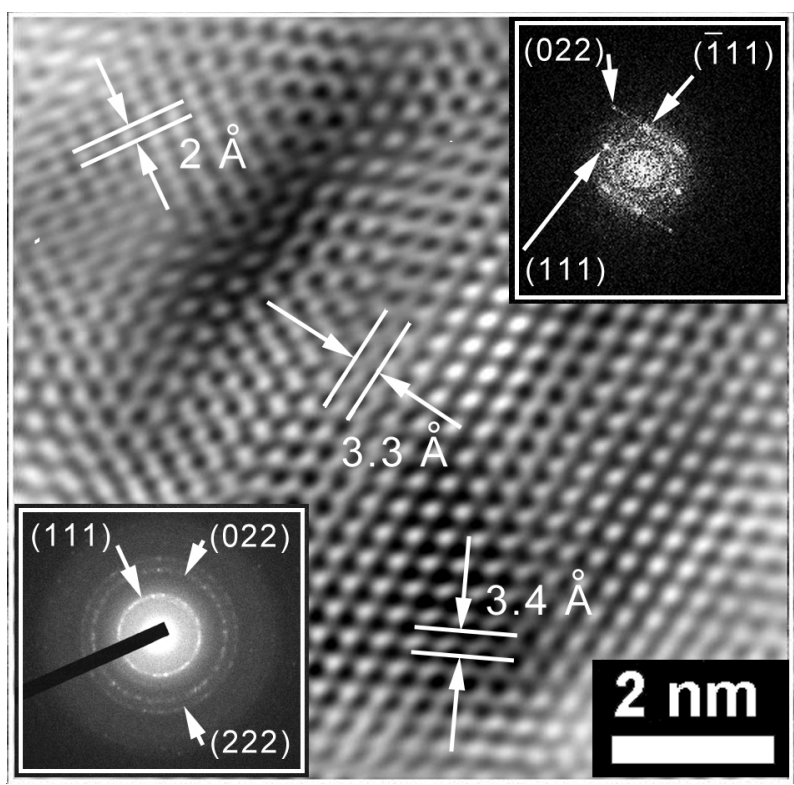

Figure 3. Enlarged (processed) HR-TEM image of a single NC, live fast Fourier transform (FFT, upper right inset) calculated from the HR-TEM image and selected area electron diffraction (SAED, bottom left inset) of the Ge-NC layer inside the $\mathrm{SiO}_{2}$ matrix. The d-spacing of the planes, as calculated by live FFT, are displayed in the HR-TEM image.

\subsection{Influence of fast neutron irradiation and annealing}

Part of Ge-NC samples were irradiated by an intensive neutron flux (with energy up to a few $\mathrm{MeV}$ ) in a research nuclear reactor with integral dose of $10^{20}$ neutrons $/ \mathrm{cm}^{2}$. As a result of this irradiation, a RS peak was decreased and broadened (curve $c$ in Fig. 1). This can be explained by the destruction of some NC and their transformation from Ge nanocrystals into amorphous clusters. However, RS peak did not disappear. This indicates that part of the Ge-NC has survived even after the high dose of destructive irradiation. The existence of remaining Ge-NC in irradiated samples was confirmed by HR-TEM image (Fig. 2c). Taking into account that the dose of irradiation was five orders of magnitude larger than the one which destroyed the devices made from bulk Ge and Si [21], one can conclude that this material shows potential for devices assigned to work in extreme conditions, such as a radiation-rich environment.

In Fig. 1, curves $d$ and $e$ show the influence of the annealing of irradiated samples on the RS spectrum. For bulk Ge, disappearance of radiation damage induced by fast neutrons is achieved after annealing at $400-450^{\circ} \mathrm{C}$ [24]. For Ge-NC, however, annealing even at $600^{\circ} \mathrm{C}$ leads only to a partial reconstruction of the RS peak (curve $d$ in Fig. 1); annealing at $800^{\circ} \mathrm{C}$ is needed for full reconstruction of the initial RS peak (inset in Fig. 1). This phenomenon can be explained by the following: In irradiated bulk Ge, the destroyed areas are surrounded by a monocrystalline matrix that serves as a seed for recrystallization. In our samples, the destroyed areas are surrounded by amorphous $\mathrm{SiO}_{2}$ which cannot promote the process of crystallization. As a result, the same temperature $\left(800^{\circ} \mathrm{C}\right)$ which was needed for the initial formation of Ge-NC after ion-implantation was still required in order to recover the crystalline structure after the fast neutron irradiation.

However, the reconstruction of crystallinity was not accompanied by the rebuilding of the initial space distribution of Ge-NC (compare Fig. $2 b$ and $2 e$ ). It is evident that the spatial distribution of nanocrystals became asymmetric - the increased average size still corresponds to the center of the projection range, while towards the $\mathrm{SiO}_{2}$ surface, the density of NC falls more rapidly.

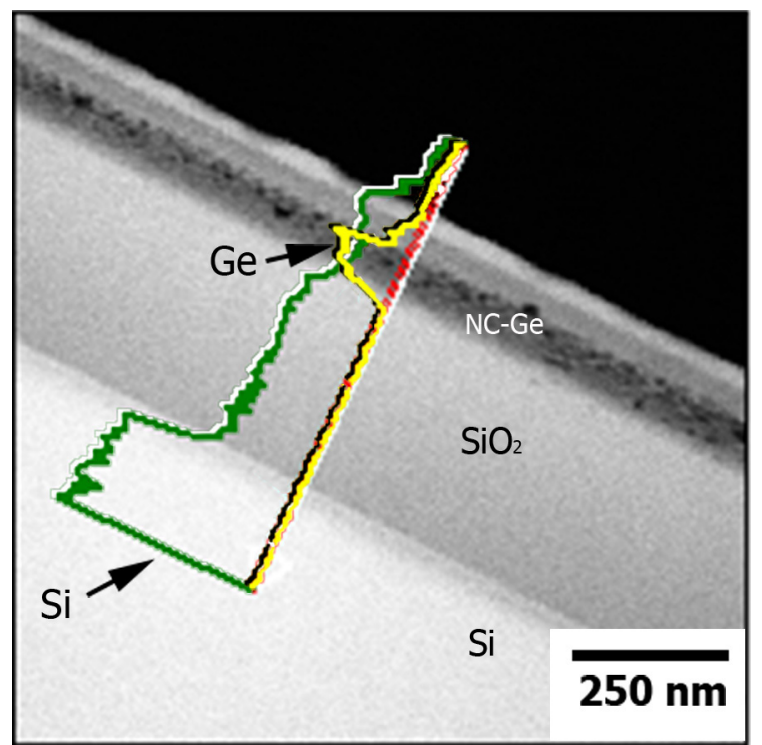

Figure 4. STEM image including an EDS line scan profile of a final sample. The curves display the abundance of elements with distance along the line (counts vs. distance along the line). The curves corresponding to elemental Ge and $\mathrm{Si}$ are shown.

The asymmetry in the space distribution of Ge-NC after neutron irradiation and annealing was also confirmed by an EDS line scan of the final sample (Fig. 4). The EDS elemental mapping (line scan profile) provides information on the chemical composition of very small volumes of material by plotting the abundance of an element with distance along a line (counts vs. distance).

Fig. 4 shows the STEM image of the cross-section of a final sample. The image clearly shows the asymmetry in distribution of $\mathrm{Ge}$ atoms inside the $\mathrm{SiO}_{2}$ matrix. 
This asymmetry can be explained by the influence of the near $\mathrm{SiO}_{2}$ surface which attracts $\mathrm{Ge}$ atoms during the final annealing. XPS measurements were performed to check the assumption regarding the enhanced diffusion of the Ge atoms towards the surface region upon the annealing of the irradiated samples. The results are shown in Fig. 5.

It is seen that in Ge-NC samples, the surface region is Ge free, while only after neutron irradiation and a second annealing at $800^{\circ} \mathrm{C}$, a peak appears at $33.4 \mathrm{eV}$, attributed to $\mathrm{Ge}$ oxides ( $\mathrm{Ge} 3 \mathrm{~d} \mathrm{GeO}_{2}$ ), and a less pronounced peak at $29.4 \mathrm{eV}$ corresponds to elemental Ge [35].

The presence of both Ge oxides and elemental Ge confirms the assumption that the asymmetry in the distribution of Ge-NC is due to diffusion of Ge atoms towards the surface during the second annealing from $600^{\circ} \mathrm{C}$ to $800^{\circ} \mathrm{C}$.

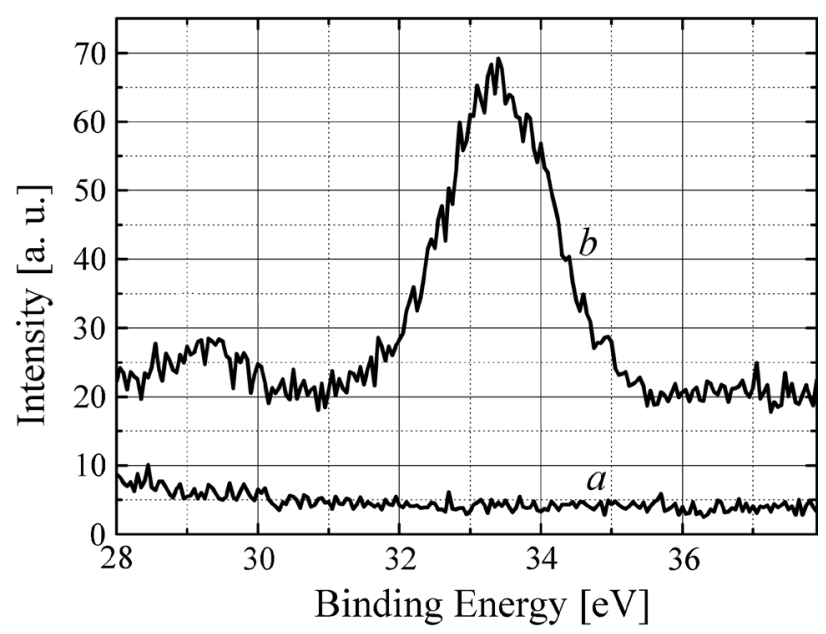

Figure 5. High resolution $X$-ray photoelectron spectroscopy (XPS) scans of Ge 3d peaks in Ge-NC (a) and final (b) samples. The $33.4 \mathrm{eV}$ peak attributed to Ge oxides $\left(\mathrm{Ge} 3 \mathrm{~d} \mathrm{GeO}_{2}\right)$, and a less pronounced peak at $29.4 \mathrm{eV}$ corresponding to elemental Ge are evident only in the final sample.

\section{Conclusions}

Investigation of Ge-NC samples embedded in an amorphous $\mathrm{SiO}_{2}$ matrix and subjected to irradiation by the neutron flux in a nuclear reactor demonstrates that part of the Ge-NC survives even after the exposure to an extremely high dose of irradiation (up to $10^{20} \mathrm{~cm}^{-2}$ ). This may indicate that if some optical or electrical properties of Ge-NC-based material are connected with existence of Ge nanocrystals, then this material may show potential for the fabrication of devices working in extreme conditions. In the case of NC, annealing of radiation damage needs higher temperatures than that in bulk Ge. After the annealing of radiation damage, crystallinity is recovered, but the space distribution of Ge-NC becomes asymmetric due to the enhanced diffusion of Ge atoms towards the $\mathrm{SiO}_{2}$ surface.

\section{Acknowledgments}

We are thankful to J. M. Lazebnik for his help in sample irradiation. We also thank M. Talianker, A. Belostotsky and Y. Fleger for technical assistance. I. S. thanks the Erick and Sheila Samson Chair of Semiconductor Technology for financial support. This work was partly supported by the Israeli-China grant 3-405.

\section{References}

[1] Y. Maeda, "Visible photoluminescence from nanocrystallite Ge embedded in a glassy $\mathrm{SiO} 2$ matrix: Evidence in support of the quantum-confinement mechanism". Phys. Rev. B vol. 51 pp.1658-1670, 1995.

[2] Y. Kanemitsu, H. Uto, Y. Masumoto and Y. Maeda, "On the origin of visible photoluminescence in nanometer-size Ge crystallites". Appl. Phys. Lett. vol. 61, pp. 2187-2189, 1992.

[3] C. J. Park, H. Y. Cho, S. Kim, S. H. Choi, R. G. Elliman, J. H. Han, C. Kim, H. N. Hwang and C. C. Hwang, "Annealing temperature dependence of capacitancevoltage characteristics in Ge-nanocrystal-based nonvolatile memory structures". J. Appl. Phys. vol. 99, pp. 36101, 2006.

[4] C. H. Tu and T. C. Chang, "Formation of germanium nanocrystals embedded in silicon-oxygen-nitride layer". Appl. Phys. Lett. vol. 89, pp. 052112, 2006.

[5] S. Duguay, J. J. Grob, A. Slaoui, Y. Le Gall and M. Amann-Liess, "Structural and electrical properties of Ge nanocrystals embedded in $\mathrm{SiO}_{2}$ by ion implantation and annealing". J. Appl. Phys. vol. 97, 104330, 2005.

[6] T. H. Ng, W. K. Chim, and W. K. Choi, “Conductancevoltage measurements on germanium nanocrystal memory structures and effect of gate electric field coupling". Appl. Phys. Lett. vol. 88, 113112, 2006.

[7] A. Kanjilal et al. "Structural and electrical properties of silicon dioxide layers with embedded germanium nanocrystals grown by molecular beam epitaxy". Appl. Phys. Lett. vol. 82, pp. 1212, 2003.

[8] S. Das, K. Das, R. K. Singha, A. Dhar and S. K. Ray, "Improved charge injection characteristics of $\mathrm{Ge}$ nanocrystals embedded in hafnium oxide for floating gate devices". Appl. Phys. Lett. vol. 91, pp. 233118, 2007.

[9] J. H. Chen, Y. Q. Wang, W. J. Yoo, Y. C. Yeo, G. Samudra, D. S. Chan, A. Y. Du and D. L. Kwong, "Nonvolatile Flash Memory Device Using Ge Nanocrystals Embedded in HfAlO High-к Tunneling and Control Oxides: Device Fabrication and Electrical Performance" IEEE transactions on electron devices, vol. 51, pp. 1840-1848, 2004.

[10] B. H. Koh, E. W. H. Kan, W. K. Chim, W. K. Choi, D. A. Antoniadis and E. A. Fitzgerald, "Traps in germanium nanocrystal memory and effect on charge retention: Modeling and experimental measurements". J. Appl. Phys. vol. 97, pp. 124305, 2005. 
[11] C. H. Tu, T. C. Chang, P. T. Liu, H. C. Liu, S. M. Sze and C. Y. Chang, "Improved memory window for Ge nanocrystals embedded in SiON layer". Appl. Phys. Lett. vol. 89, pp. 162105, 2006.

[12] O. González-Varona, B. Garrido, S. Cheylan, A. Pérez-Rodríguez, A. Cuadras and J. R. Morante, "Control of tunnel oxide thickness in Si-nanocrystal array memories obtained by ion implantation and its impact in writing speed and volatility ". Appl. Phys. Lett. vol. 82, pp. 2151, 2003.

[13] Q. Wan, N. L. Zhang, W. L. Liu, C. L. Lin and T. H. Wang, "Memory and negative photoconductivity effects of Ge nanocrystals embedded in $\mathrm{ZrO}_{2} / \mathrm{Al}_{2} \mathrm{O}_{3}$ gate dielectrics". Appl. Phys. Lett. vol. 83, pp. 138-140, 2003.

[14] S. Levy, I. Shlimak, A. Chelly and Z. Zalevsky, "Influence of Ge nanocrystals and radiation defects on C-V characteristics in Si-MOS structures". Physica B vol. 404, pp. 5189-5191, 2009.

[15] P. W. Li, W. M. Liao, D. M. T. Kuo, S. W. Lin, P. S. Chen, S. C. Lu and M. J. Tsai, "Fabrication of a germanium quantum-dot single-electron transistor with large Coulomb-blockade oscillations at room temperature". Appl. Phys. Lett. vol. 85, pp. 1532-1534, 2004.

[16] J. M. Shieh, Y. F. Lai, W. X. Ni, H. C. Kuo, C. Y. Fang, J. Y. Huang and C. L. Pan, "Enhanced photoresponse of a metal-oxide-semiconductor photodetector with silicon nanocrystals embedded in the oxide layer". Appl. Phys. Lett. vol. 90, pp. 051105, 2007.

[17] X. B. Lu, P. F. Lee and J. Y. Dai, "Synthesis and memory effect study of Ge nanocrystals embedded in $\mathrm{LaAlO}_{3}$ high-k dielectrics". Appl. Phys. Lett. vol. 86, pp. 203111, 2005.

[18] M. Kanoun, A. Souifi, T. Baron and F. Mazen, "Electrical study of Ge-nanocrystal-based metaloxide-semiconductor structures for p-type nonvolatile memory applications". Appl. Phys. Lett. vol. 84, pp. 5079, 2004.

[19] M. C. Ridgway et al. "Preferential amorphisation of Ge nanocrystals in a silica matrix". Nuclear Instruments and Methods in Physics Research B, vol. 242, pp. 121-124, 2006.

[20] See edited by J. M. Meese, Neutron Transmutation Doping in Semiconductors, New York: Plenum Press, 1979, and references there in.

[21] H. Ohyama, J. Vanhellemont, Y. Takami, K. Hayama, H. Sunaga,J. Poortmans, M. Caymax and P. Clauws, "Germanium content dependence of radiation damage in strained Si1-x Gex epitaxial devices". IEEE Transaction on Nuclear Science, vol. 41 (6), pp. 2437, 1994.

[22] G. C. Messenger and M. S. Ash, The effects of radiation on electronic systems. 2nd ed. New York: Van Nostrand Reinhold, 1992.
[23] I. S. Shlimak, "Neutron transmutation doping in semiconductors: science and applications". Solid State, vol. 41, pp. 716-719, 1999.

[24] I. Shlimak, A. N. Ionov, R. Rentzsch, J. M. Lazebnik, "On the doping of isotopically controlled germanium by nuclear transmutation with a high concentration of shallow donor impurities". Semicond. Sci. Technol. vol.11, pp. 1826, 1996.

[25] Y. Hu et al. "The Raman spectroscopy of neutron transmutation doping isotope ${ }^{74}$ Germanium nanocrystals embedded in $\mathrm{SiO}_{2}$ matrix". Solid State Communications, vol. 141, pp. 514-518, 2007.

[26] S. Dun et al. "Effect of As doping on the photoluminescence of nanocrystalline ${ }^{74} \mathrm{Ge}$ embedded in $\mathrm{SiO}_{2}$ matrix". Journal of Luminescence, vol. 128, pp. 1363-1368, 2008.

[27] Y. Hu et al. "Neutron transmutation doping effect on the optical property of germanium nanocrystals". Scripta Materialia, vol. 61, pp. 970-973, 2009.

[28] Q. Chen, T. Lu, M. Xu, C. Meng, Y. Hu, K. Sun, and I. Shlimak, "Fabrication of uniform Ge-nanocrystals embedded in amorphous $\mathrm{SiO}_{2}$ films using Ge-ion implantation and neutron irradiation methods". Appl. Phys. Lett. vol. 98, pp. 073103, 2011.

[29] M. Fujii, S. Hayashi and K. Yamamoto, "Growth of Ge microcrystals in $\mathrm{SiO}_{2}$ thin film matrices: A Raman and Electron Microscopic Study". Japanese Journal of Applied Physics, vol. 30, pp. 687-694, 1991.

[30] M. Fujii, S. Hayashi and K. Yamamoto, "Raman scattering from quantum dots of Ge embedded in $\mathrm{SiO}_{2}$ thin films". Appl. Phys. Lett. vol. 57, pp. 2692-2694, 1990.

[31] U. Serincan, G. Kartopu, A. Guennes, T. G. Finstad, R. Turan, Y. Ekinci and S. C. Bayliss, "Characterization of Ge nanocrystals embedded in $\mathrm{SiO}_{2}$ by Raman spectroscopy" Semicond. Sci. Technol. vol. 18, pp. 247, 2004.

[32] J. F. Zigler, http://www.srim.org/index.htm, (Copyright 1984-2006).

[33] I. D. Sharp et. al, "Mechanism of stress relaxation in Ge nanocrystals embedded in $\mathrm{SiO}_{2}{ }^{\prime \prime}$. Appl. Phys. Lett. vol. 86, pp. 86, 063107, 2005 .

[34] H. Richter, Z. P. Wang and L. Ley, "The one phonon Raman spectrum in microcrystalline silicon". Solid State Communication. vol. 39, pp. 625-629, 1981.

[35] A. K. Dutta, “Visible photoluminescence from Ge nanocrystal embedded into a $\mathrm{SiO}_{2}$ matrix fabricated by atmospheric pressure chemical vapor deposition". Appl. Phys. Lett. vol. 68, pp. 1189, 1996. 